Pratibhamoy Das, Volker Mehrmann

\title{
Numerical solution of singularly perturbed convection-diffusion-reaction problems with two small
}

\section{Article, Preprint Version}

This version is available at https://depositonce.tu-berlin.de.

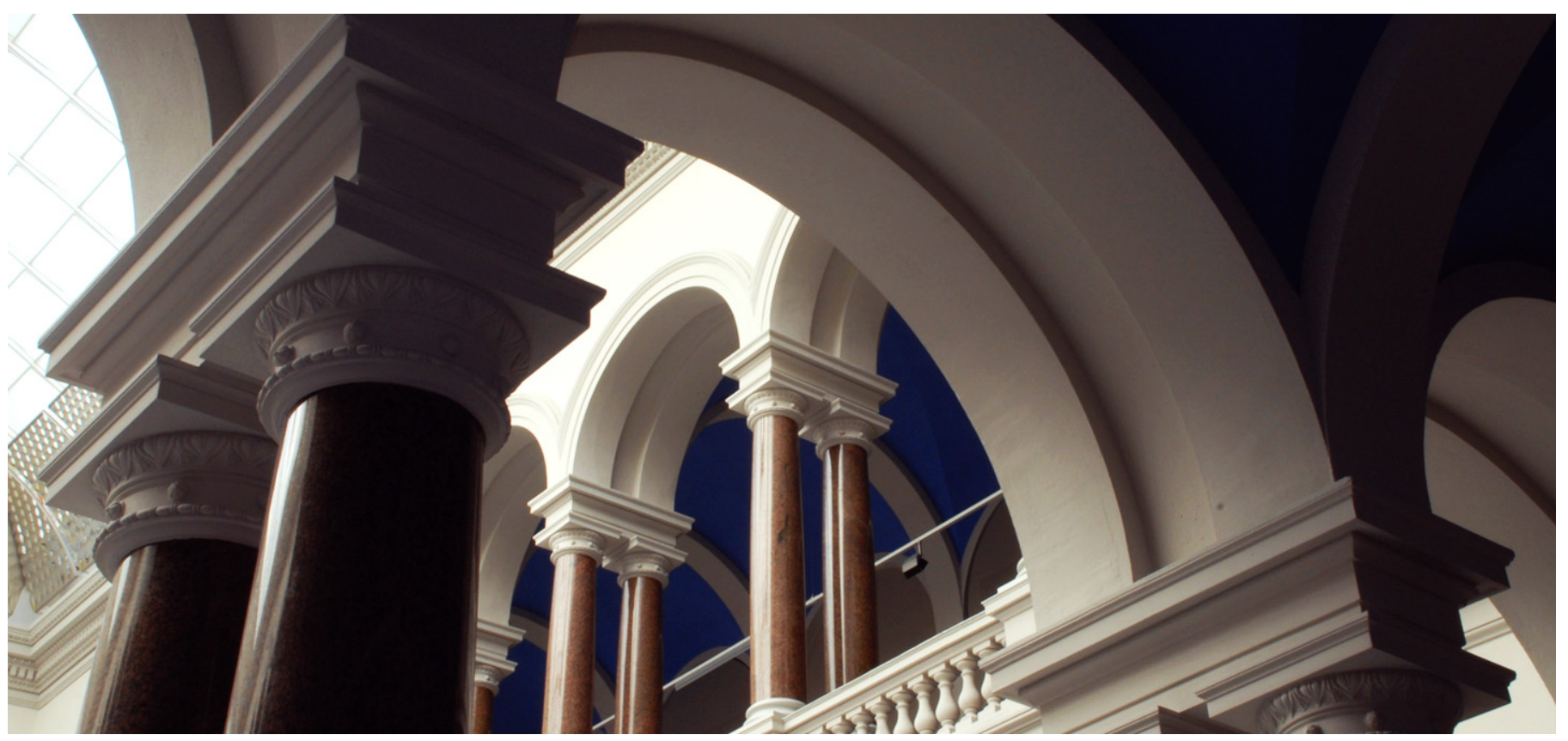

\section{Suggested Citation}

Numerical solution of singularly perturbed convection-diffusion-reaction problems with two small. - BIT Numerical Mathematics. - ISSN: 1572-9125 (online), 0006-3835 (print). - Published online 09 July 2015. DOI:10.1007/s10543-015-0559-8.

The final publication is available at Springer via http://dx.doi.org/10.1007/s10543-015-0559-8. 


\title{
Numerical solution of singularly perturbed convection-diffusion-reaction problems with two small parameters
}

\author{
Pratibhamoy Das * $\quad$ Volker Mehrmann *
}

January 6, 2016

\begin{abstract}
This paper discusses the numerical solution of 1-D convection-diffusion-reaction problems that are singularly perturbed with two small parameters using a new mesh-adaptive upwind scheme that adapts to the boundary layers. The meshes are generated by the equidistribution of a special positive monitor function. Uniform, parameter independent convergence is shown and holds even in the limit that the small parameters are zero. Numerical experiments are presented that illustrate the theoretical findings, and show that the new approach has better accuracy compared with current methods.
\end{abstract}

Key words: Parabolic partial differential equation, convection-diffusion-reaction problem, upwind scheme, adaptive mesh, mesh equidistribution, two parameter singular perturbation problem, uniform convergence.

AMS Subject Classification: 65L06, 65M12, 65M50

\section{Introduction}

The numerical solution of singularly perturbed perturbed convection-diffusion-reaction is an important problem in many applications [20, 21]. The presence of small perturbation parameters makes the numerical analysis difficult for these problems, see e.g. [6, 22, 27, 28]. To obtain efficient methods, adaptive mesh generation in finite element or finite difference methods is necessary, since for singularly perturbed problems boundary layers arise in the solution that can only be resolved by very fine meshes in these layers. Singular perturbation problems often have as limiting case differential-algebraic equations, which require consistency of the boundary conditions to avoid jumps in the solution. For differential-algebraic equations the theoretical and numerical analysis have been studied in detail in recent years, see e.g. [3, 13, 19]. In order to adapt to the boundary layers, the number of mesh points has to proportional to the inverse power of perturbation parameters. Hence, to avoid uniformly fine meshes in many approaches, see e.g. [4, 16, the use of a priori refined meshes is suggested to capture the solution behavior in the boundary layers numerically. For steady state problems, see e.g. [16, 18, 24, the a priori defined meshes lead to a successful approach even for problems involving two singular perturbation parameters. However, if a priori information about the solution is not available, then an automatically generated adaptive mesh is desirable.

\footnotetext{
*Institut für Mathematik, Technische Universität Berlin, Str. des 17. Juni 136, D-10623 Berlin, Fed. Rep. Germany, \{pratibha, mehrmann\}@math.tu-berlin.de
} 
In this paper we propose an adaptive finite difference method for the numerical solution of 1-D parabolic convection-diffusion-reaction initial-boundary value problems with two small parameters, where a priori information about the solution is not needed. The space adaptive mesh within the numerical integration of the dynamics is constructed by moving a fixed number of mesh points in a way which can automatically detect the layers.

We consider as a model problem the following 1-D singularly perturbed initial-boundaryvalue problem (IBVP) on a domain $\Omega=\Omega_{x} \times(0, T]$ with $\Omega_{x}=(0,1)$,

$$
\begin{gathered}
\frac{\partial u}{\partial t}-\varepsilon \frac{\partial^{2} u}{\partial x^{2}}-\mu b(x, t) \frac{\partial u}{\partial x}+c(x, t) u=f(x, t), \quad(x, t) \in \Omega, \\
u(x, 0)=u_{0}(x), x \in \bar{\Omega}_{x}, u(0, t)=u(1, t)=0, t \in[0, T],
\end{gathered}
$$

and we use the abbreviation

$$
L u(x, t):=-\varepsilon u_{x x}-\mu b(x, t) u_{x}+c(x, t) u,
$$

where $u_{x x}=\frac{\partial^{2} u}{\partial x^{2}}, u_{x}=\frac{\partial u}{\partial x}$, and $u_{t}=\frac{\partial u}{\partial t}$.

Here $0 \leq \varepsilon \ll 1$ and $0 \leq \mu \ll 1$ are two small parameters, and the coefficient functions $b(x, t), c(x, t)$ and $f(x, t)$ are assumed to be sufficiently smooth and to satisfy constraints $\beta_{1}>b(x, t)>\beta_{2}>0$ and $c(x, t) \geq 1$ on the closure $\bar{\Omega}_{x}=[0,1]$. To simplify our analysis, we assume that the parameters $\varepsilon$ and $\mu$ satisfy the relation $\mu^{2} \leq \varepsilon$, but similar results can be obtained without this extra assumption. Under sufficient smoothness and suitable compatibility conditions on the data, the IBVP (1.1) has a unique solution $u(x, t)$, which exhibits boundary layers in the neighborhood of both spacial boundaries $x=0$ and $x=1$, see [16].

For the adaptive mesh generation we will employ the idea of equidistribution, see e.g. [15]. A space-mesh $\Omega_{x}^{n} \equiv\left\{0=x_{0}^{n}<x_{1}^{n}<\cdots<x_{M}^{n}=1\right\}$ in the $n$-th time-step of a space-time finite-difference method is said to be equidistributed, if

$$
\int_{x_{m-1}^{n}}^{x_{m}^{n}} \Phi\left(s, u\left(s, t_{n}\right)\right) d s=\frac{1}{M} \int_{0}^{1} \Phi\left(s, u\left(s, t_{n}\right)\right) d s, \quad m=1, \ldots, M,
$$

where $\Phi(s, u(s, t))>0$ is a given monitor function. Mesh equidistribution can be viewed as a mapping $x^{n}=x^{n}(\xi)$ from a computational coordinate $\xi \in[0,1]$ to the physical coordinate $x^{n} \in \bar{\Omega}_{x}$, defined by

$$
\int_{0}^{x^{n}(\xi)} \Phi\left(s, u\left(s, t_{n}\right)\right) d s=\xi \int_{0}^{1} \Phi\left(s, u\left(s, t_{n}\right)\right) d s .
$$

Adaptive moving mesh methods based on the idea of equidistribution were proposed in [2] for steady state singularly perturbed convection-diffusion problems and 14 for non-stationary partial differential equations. Parabolic singularly perturbed initial boundary problems which only involve a diffusion parameter were considered in [11, 12, 22, 23], where several kind of numerical techniques were developed for uniform convergence. In [11, 12] a curvature based and in [25], an arc-length based monitor function is suggested to generate the boundary layer-adapted meshes for parabolic problems. Numerical methods using two-step backward difference time discretization can be found in [7, 8, 9], see also [30] for a detailed survey on 
time dependent singularly perturbed problems and appropriate efficient numerical methods. In the present work, we consider a monitor function for a parabolic initial-boundary-value problem with small convection as well as diffusion parameters that generalizes the work of [11].

The outline of the paper is as follows. Section 2 introduces a decomposition of the analytical solution into its regular (interior) and singular (layer) components, and provides a priori bounds for the solution and its derivatives. The fully discrete problem and the behavior of the equidistributed meshes corresponding to (1.1) are also addressed. In Section 3 we show the first-order, parameter uniform, space-time convergence and derive a stability estimate for the discrete problem. Finally, in Section 4 we present several numerical experiments that illustrate the theoretical results.

Throughout this paper $C$ denotes a generic positive constant independent of $\varepsilon, \mu, N$, and $M$, which can take different values at different places. We use $\|\phi(x)\|_{D}=\max _{\eta \in D}|\phi(\eta)|$ to define the infinity norm for a function $\phi$ defined on a domain $D$. When the domain is obvious, or of no particular significance, we simply use $\|\cdot\|$ instead of $\|\cdot\|_{D}$. For any domain $\Omega$, we denote $\bar{\Omega}$ as the closure of $\Omega$ and by $\partial \Omega$ its boundary. For a function $U$ that is defined on a discrete mesh $x_{0}, \ldots, x_{M}$ in $\Omega_{x}^{n}$, we define $\bar{U}$ as the piecewise linear interpolant, i.e. $\bar{U}$ is continuous on $\overline{\Omega_{x}^{n}}$, and linear on each $\left[x_{i-1}^{n}, x_{i}^{n}\right]$ and satisfies $\bar{U}\left(x_{i}\right)=U_{i}$ for each $i=0, \cdots, M$.

\section{The space-time continuous problem}

In this section, we recall some analytical properties of the solution $u(x, t)$ of (1.1). For this, we decompose the analytic solution $u(x, t)$ into two components, a regular component $v(x, t)$ which characterizes the solution behavior outside the boundary layers, and a singular component $w(x, t)$ which characterizes the solution behavior inside the boundary layers such that $u(x, t)=v(x, t)+w(x, t)$ where the regular component $v(x, t)$ satisfies

$$
\frac{\partial v}{\partial t}(x, t)+L v=f, \quad v(x, 0)=u(x, 0), \quad \text { for } \quad(x, t) \in \Omega_{x} \times(0, T],
$$

with the required boundary conditions at the end points. We further decompose the singular component $w$ into the sum of its left and right singular part $w=w_{l}+w_{r}$, associated with left and right boundary layers, such that

$$
\begin{aligned}
& \frac{\partial w_{l}}{\partial t}(x, t)+L w_{l}(x, t)=0, \quad(x, t) \in \Omega, \\
& w_{l}(x, 0)=0, w_{l}(1, t)=0, w_{l}(0, t)=u(0, t)-v(0, t),
\end{aligned}
$$

and

$$
\begin{aligned}
& \frac{\partial w_{r}}{\partial t}(x, t)+L w_{r}(x, t)=0,(x, t) \in \Omega \\
& w_{r}(x, 0)=0, w_{r}(0, t)=0, w_{r}(1, t)=u(1, t)-v(1, t) .
\end{aligned}
$$

For the analysis, we first perform a semi-discretization of the parabolic IBVP (1.1) in time by using the implicit Euler method with uniform time-steps $\Delta t=T / N$ in the time-interval $[0, T]$. This gives a time-mesh $\Omega_{t}^{N} \equiv\left\{t_{n}=n \Delta t, n=0, \cdots, N\right\}$. Note that the presented

results are easily extended to other one-step time discretization methods including those with variable time-mesh. 
In the $(n+1)$-st time step, the semi-discrete solution $u^{n+1}(x)$ of the IBVP 1.1 will satisfy

$$
\begin{aligned}
& u^{0}=u(x, 0)=u_{0}(x), x \in \Omega_{x}, \\
& \frac{1}{\Delta t}\left[u^{n+1}(x)-u^{n}(x)\right]-\varepsilon u_{x x}^{n+1}(x)-\mu b^{n+1}(x) u_{x}^{n+1}(x)+c^{n+1}(x) u^{n+1}(x)=f\left(x, t_{n+1}\right), \\
& u^{n+1}(0)=u^{n+1}(1)=0,
\end{aligned}
$$

where $b^{n+1}(x):=b\left(x, t_{n+1}\right)$ and $c^{n+1}(x):=c\left(x, t_{n+1}\right)$. We can rewrite 2.4 as a differential equation in the space variable $x$,

$$
\begin{aligned}
& u^{0}=u(x, 0)=u_{0}(x), x \in \Omega_{x}, \\
& -\varepsilon u_{x x}^{n+1}(x)-\mu b^{n+1}(x) u_{x}^{n+1}(x)+a^{n+1}(x) u^{n+1}(x)=g^{n+1}(x), \\
& u^{n+1}(0)=u^{n+1}(1)=0,
\end{aligned}
$$

where $a^{n+1}(x):=c^{n+1}(x)+1 / \Delta t$ and $g^{n+1}(x):=f\left(x, t_{n+1}\right)+u^{n}(x) / \Delta t$.

It is well-known [12] that the implicit Euler method ensures the stability of the solution of 2.5 and if we denote the local truncation error in the $(n+1)$-st time-step at a point $\left(x, t^{n+1}\right) \in \Omega_{x} \times(0, T]$ by $\tau_{n+1}(x):=u\left(x, t^{n+1}\right)-\tilde{u}^{n+1}(x)$, where $\tilde{u}^{n+1}(x)$ is the numerical solution of

$$
\begin{aligned}
& -\varepsilon \tilde{u}_{x x}^{n+1}(x)-\mu b^{n+1}(x) \tilde{u}_{x}^{n+1}(x)+a^{n+1}(x) \tilde{u}^{n+1}(x)=g^{n+1}(x), x \in \Omega_{x}, \\
& \tilde{u}^{n+1}(0)=\tilde{u}^{n+1}(1)=0,
\end{aligned}
$$

then we have the following consistency result.

Lemma 2.1 [16] The solution $u(x, t)$ of (1.1) and its time derivative $u_{t}(x, t)$ are bounded in $\bar{\Omega}$, independent of $\epsilon, \mu, N, M$. The local error $\tau_{n+1}(x)=u\left(x, t^{n+1}\right)-\tilde{u}^{n+1}(x)$ satisfies

$$
\left\|\tau_{n+1}\right\| \leq C(\Delta t)^{2}
$$

and the global error satisfies

$$
\max _{n+1 \leq T / \Delta t}\left\|u\left(x, t_{n+1}\right)-\tilde{u}^{(n+1)}(x)\right\| \leq C \Delta t,
$$

with a constant $C$ that is independent of $\varepsilon, \mu, x$, and $t$.

Lemma 2.1 implies that the semi-discretized scheme is first-order uniformly convergent in time.

For the further analysis, we use the characteristic equation

$$
-\varepsilon \psi^{2}(x)-\mu b^{n+1}(x) \psi(x)+a^{n+1}(x)=0
$$

associated with (2.6), which has two real roots $\psi_{l}(x)<0$ and $\psi_{r}(x)>0$. The quantity $\psi_{l}(x)$ is associated with the boundary layer at $x=0$ and $\psi_{r}(x)$ with the boundary layer at $x=1$. Let us define the bounds

$$
\Psi_{l}:=-\max _{x \in \Omega_{x}} \psi_{l}(x)>0 \quad \text { and } \quad \Psi_{r}:=\min _{x \in \Omega_{x}} \psi_{r}(x)>0 .
$$

The following lemma gives upper bounds for the regular and singular components of the solution. 
Lemma 2.2 [16] The numerical solution $\tilde{u}^{n+1}(x)$ of 2.5) can be decomposed, as in (2.1)(2.2), into a component $\tilde{v}(x)$ associated with the regular part, as well as left and right components $\tilde{w}_{l}(x)$ and $\tilde{w}_{r}(x)$, associated with the boundary layers, respectively, such that

$$
\left|\tilde{v}^{k}(x)\right| \leq C, \quad\left|\tilde{w}_{l}^{k}(x)\right| \leq C \Psi_{l}^{k} \exp \left(-p \Psi_{l} x\right), \text { and }\left|\tilde{w}_{r}^{k}(x)\right| \leq C \Psi_{r}^{k} \exp \left(-p \Psi_{r}(1-x)\right),
$$

with $0 \leq k \leq q$, for a prescribed $q$ and a fixed number $0<p<1$. In particular, one has the following derivative bound

$$
\left|\frac{\partial^{k} \tilde{u}(x)}{\partial x^{k}}\right| \leq C\left(1+\Psi_{l}^{k} \exp \left(-p \Psi_{l} x\right)+\Psi_{r}^{k} \exp \left(-p \Psi_{r}(1-x)\right)\right), \quad \text { for } \quad 0 \leq k \leq q,
$$

where the constant $C$ is independent of $\varepsilon, \mu$.

\subsection{Finite difference discretization in space}

In this subsection we consider the finite difference approximation of (1.1) on a nonuniform space-mesh $\Omega_{x}^{n} \equiv\left\{0=x_{0}^{n}<x_{1}^{n}<\cdots<x_{M}^{n}=1\right\}$, where $n$ denotes the time level and the step-sizes are defined by $h_{m+1}^{n}=x_{m+1}^{n}-x_{m}^{n}, m=0, \cdots, M-1$. For a discrete space-time mesh function $V_{m}^{n}:=v\left(x_{m}, t_{n}\right)$, we introduce the central difference operator

$$
\Delta_{x}^{2} V_{m}^{n}:=\frac{\left(D_{x}^{+} V_{m}^{n}-D_{x}^{-} V_{m}^{n}\right)}{\left(h_{m}^{n}+h_{m+1}^{n}\right) / 2}, \text { where } D_{x}^{+} V_{m}^{n}:=\frac{V_{m+1}^{n}-V_{m}^{n}}{h_{m+1}^{n}} \text { and } D_{x}^{-} V_{m}^{n}=\frac{V_{m}^{n}-V_{m-1}^{n}}{h_{m}^{n}}
$$

For problem (2.4) we use an upwind scheme of the form

$$
\begin{aligned}
& \left(I+\Delta t L^{M}\right) U_{m}^{n+1}=U_{m}^{n}+\Delta t f\left(x_{m}^{n+1}, t_{n+1}\right), m=1, \cdots M-1, \\
& U_{0}^{n+1}=U_{M}^{n+1}=0
\end{aligned}
$$

where $L^{M}$ is the discrete operator associated to the continuous operator $L$ in $(1.2)$, given by

$$
L^{M} U_{m}^{n+1} \equiv-\varepsilon \Delta_{x}^{2} U_{m}^{n+1}-\mu b_{m}^{n+1} D_{x}^{+} U_{m}^{n+1}+c_{m}^{n+1} U_{m}^{n+1},
$$

with

$$
b_{m}^{n+1}:=b\left(x_{m}^{n+1}, t_{n+1}\right), c_{m}^{n+1}:=c\left(x_{m}^{n+1}, t_{n+1}\right) .
$$

We again rearrange $(2.9)$ to the form

$$
\begin{aligned}
& r_{m, n+1}^{-} U_{m-1}^{n+1}+r_{m, n+1}^{*} U_{m}^{n+1}+r_{m, n+1}^{+} U_{m+1}^{n+1}=g_{m}^{n}, m=1, \cdots M-1, \\
& U_{0}^{n+1}=U_{M}^{n+1}=0,
\end{aligned}
$$

where the coefficients are given by

$$
\begin{aligned}
r_{m, n+1}^{-} & :=\frac{-2 \varepsilon \Delta t}{h_{m}^{n+1}\left(h_{m}^{n+1}+h_{m+1}^{n+1}\right)}, r_{m, n+1}^{+}:=\frac{-2 \varepsilon \Delta t}{h_{m+1}^{n+1}\left(h_{m}^{n+1}+h_{m+1}^{n+1}\right)}-\frac{\mu \Delta t b_{m}^{n+1}}{h_{m+1}^{n+1}} \\
r_{m, n+1}^{*} & :=1+\Delta t c_{m}^{n+1}-r_{m, n+1}^{-}-r_{m, n+1}^{+}, g_{m}^{n}:=\bar{U}\left(x_{m}^{n+1}, t_{n}\right)+\Delta t f\left(x_{m}^{n+1}, t_{n+1}\right)(2.21
\end{aligned}
$$

where $\bar{U}$ is the linear interpolant of $U$. 
Similarly, the discretized problem for the regular component will be constructed via

$$
\begin{aligned}
& \left(1+\Delta t c_{m}^{n+1}\right) V_{m}^{n+1}=\bar{V}\left(x_{m}^{n+1}, t_{n}\right)+\Delta t f\left(x_{m}^{n+1}, t_{n+1}\right), m=1, \cdots M-1, \\
& \bar{V}^{0}(x)=0
\end{aligned}
$$

where $\bar{V}$ is the linear interpolant of $V$.

From the a priori analysis [24] it can be observed that the boundary layers arise from the left and right singular components of the solution. This motivates us to consider a particular monitor function that involves derivatives of the layer parts, and is given by

$$
\Phi\left(x, u\left(x, t_{n+1}\right)\right)=\alpha^{n+1}+\left|w_{x x}\left(x, t_{n+1}\right)\right|^{1 / 2} .
$$

Here, $\alpha^{n+1}$ is a positive constant that is introduced so that the mesh does not degenerate inside the boundary layers. Similar monitor functions involving the derivatives of a singular component of the solution have been introduced also in convection dominated problems, see e.g. [2, 5].

To approximate the second derivative $w_{x x}$, we use the leading terms in the Taylor expansion of $w$ (see Lemma 2.2 which implies that

$$
\left|w_{x x}\left(x, t_{n+1}\right)\right| \approx\left\{\begin{array}{l}
\kappa_{l} \Psi_{l}^{2} \exp \left(-p \Psi_{l} x\right), \quad x \in[0,1 / 2] \\
\kappa_{r} \Psi_{r}^{2} \exp \left(-p \Psi_{r}(1-x)\right), \quad x \in(1 / 2,1]
\end{array}\right.
$$

where $\kappa_{l}$ and $\kappa_{r}$ are constants that are independent of $\varepsilon, \mu$ and $x$. Hence, we obtain an approximation of the monitor function given

$$
\int_{0}^{1}\left|w_{x x}\left(x, t_{n+1}\right)\right|^{1 / 2} d x \equiv K \approx 2 p^{-1}\left(| \kappa _ { l } | ^ { 1 / 2 } \left(1-\exp \left(-p \Psi_{l} / 4\right)+\left|\kappa_{r}\right|^{1 / 2}\left(1-\exp \left(-p \Psi_{r} / 4\right)\right) .\right.\right.
$$

Substituting this approximation of $w_{x x}\left(x, t_{n+1}\right)$ into the mapping 1.4 defining the equidistribution principle, for the left side of the interval with $x^{n+1}(\xi) \leq 1 / 2$, we obtain

$$
\xi\left(\frac{\alpha^{n+1}}{K}+1\right)=\alpha^{n+1} \frac{x^{n+1}(\xi)}{K}+\frac{2\left|\kappa_{l}\right|^{1 / 2}}{p K}\left[1-\exp \left(-\frac{p \Psi_{l} x^{n+1}(\xi)}{2}\right)\right]
$$

and for the right side of the interval with $x^{n+1}(\xi) \geq 1 / 2$, the mapping 1.4 leads to

$$
(1-\xi)\left(\frac{\alpha^{n+1}}{K}+1\right)=\alpha^{n+1} \frac{\left(1-x^{n+1}(\xi)\right)}{K}+\frac{2\left|\kappa_{r}\right|^{1 / 2}}{p K}\left[1-\exp \left(-\frac{\left(1-x^{n+1}(\xi)\right)}{2} p \Psi_{r}\right)\right] .
$$

Considering (1.4 as a mapping from the physical non-uniform coordinates $\left\{x_{m}^{n+1}\right\}_{m=0}^{M}$ to the computational coordinates of a uniform meshes $\left\{\xi_{m}^{n+1}=m / M\right\}_{m=0}^{M}$, from 2.15 for $x_{m}^{n+1} \leq 1 / 2$ we obtain

$$
\frac{\alpha^{n+1} x_{m}^{n+1}}{K}+K_{l}\left[1-\exp \left(\frac{-p \Psi_{l} x_{m}^{n+1}}{2}\right)\right]=\frac{m}{M}\left(\frac{\alpha^{n+1}}{K}+1\right)
$$

and from 2.16 for $x_{m}^{n+1} \geq 1 / 2$, we obtain

$$
\frac{\alpha^{n+1}\left(1-x_{m}^{n+1}\right)}{K}+K_{r}\left[1-\exp \left(\frac{-\left(1-x_{m}^{n+1}\right) p \Psi_{r}}{2}\right)\right]=\left(1-\frac{m}{M}\right)\left(\frac{\alpha^{n+1}}{K}+1\right)
$$


where $K_{l}=2\left|\kappa_{l}\right|^{1 / 2} / p K$ and $K_{r}=2\left|\kappa_{r}\right|^{1 / 2} / p K$.

From this construction, it is clear that the adaptively equidistributed mesh that is generated in this way will satisfy both the nonlinear algebraic equations (2.17) and (2.18). The following lemma provides the structure of the mesh distribution and also presents a choice for $\alpha^{n+1}$.

Lemma 2.3 Suppose that a nonuniform mesh is generated with $\alpha^{n+1}=K$ in (2.17) and (2.18). Then the mesh points are distributed as

$$
x_{z_{l}}^{n+1}<\frac{2}{p \Psi_{l}} \ln (M)<x_{z_{l}+1}^{n+1}, x_{z_{r}-1}^{n+1}<1-\frac{2}{p \Psi_{r}} \ln (M)<x_{z_{r}}^{n+1},
$$

where $z_{l}, z_{r}$ denote the integer parts of

$$
\frac{K_{l}}{2}(M-1)+\frac{2 M \ln (M)}{p \Psi_{l}}, M-\frac{1}{2}\left(K_{r}(M-1)+\frac{2 M \ln (M)}{p \Psi_{r}}\right)+1,
$$

respectively.

Proof. The assertions follow by setting $\alpha^{n+1}=K$ and $x_{m}^{n+1}=\frac{2}{p \Psi_{l}} \ln (M)$ in 2.17), and evaluating for $m$, and analogous for $x_{m}^{n+1}=\frac{2}{p \Psi_{r}} \ln (M)$ in 2.18 .

The next lemma provides insight in the resulting mesh spacing.

Lemma 2.4 Inside the boundary layers, the mesh satisfies the bounds

$$
\begin{aligned}
\Psi_{l} h_{m}^{n+1} & <C, m=1, \cdots, z_{l}, \\
\Psi_{r} h_{m}^{n+1} & <C, m=z_{r}+1, \cdots, M .
\end{aligned}
$$

Proof. The bounds for the mesh spacing can be obtained by using upper and lower bounds on the location of $x_{m}^{n+1}$. Consider two points $\bar{x}_{m}^{n+1}>x_{m}^{n+1}$ and $\underline{x}_{m}^{n+1}<x_{m}^{n+1}$. Then, from 2.17) we obtain that $\bar{x}_{m}^{n+1}$ satisfies

$$
\exp \left(-\frac{p \Psi_{l} \bar{x}_{m}^{n+1}}{2}\right)=1-\frac{2 m}{M K_{l}}
$$

which implies that $\bar{x}_{m}^{n+1}=-\frac{2}{p \Psi_{l}} \log \left(1-\frac{2 m}{M K_{l}}\right)$. Again, from 2.17) we have that $\underline{x}_{m}^{n+1}$ satisfies

$$
\exp \left(-\frac{p \Psi_{l} \underline{x}_{m}^{n+1}}{2}\right)-\frac{\bar{x}_{m}^{n+1}}{K_{l}} \leq 1-\frac{2 m}{M K_{l}}
$$

which implies that

$$
-\frac{p \Psi_{l} \underline{x}_{m}^{n+1}}{2} \leq \log \left(1-\frac{2 m}{M K_{l}}+\frac{\bar{x}_{m}^{n+1}}{K_{l}}\right)
$$

Therefore,

$$
\underline{x}_{m}^{n+1} \geq-\frac{2}{p \Psi_{l}} \log \left(1-\frac{1}{K_{l}}\left(\frac{2 m}{M}+\frac{2}{p \Psi_{l}} \log \left(1-\frac{2 m}{M K_{l}}\right)\right)\right),
$$


and, hence

$$
\begin{aligned}
h_{m}^{n+1} & <\bar{x}_{m}^{n+1}-\underline{x}_{m-1}^{n+1} \\
& \leq \frac{2}{p \Psi_{l}}\left[\log \left(1+\frac{2}{M K_{l}-2 m}+\frac{2 M K_{l}^{2}}{p \Psi_{l}\left(M K_{l}-2 m\right)} \log \left(\frac{M K_{l}}{M K_{l}-2 m+2}\right)\right)\right] \\
& \leq \frac{C}{\Psi_{l}} .
\end{aligned}
$$

The other bound for $h_{m}^{n+1}$ follows analogously. $\quad \square$

One also obtains the following upper bound for the mesh spacing in the whole domain.

Lemma 2.5 The equidistribution of monitor function (2.14) generates a mesh that satisfies

$$
h_{m}^{n+1} \leq C M^{-1}, m=1, \cdots, M .
$$

Proof. It is easy to see that the monitor function (2.14), satisfies $\Phi\left(x, u\left(x, t_{n+1}\right)\right) \geq \alpha^{n+1}=$ $K$. Again, the estimates for the derivative of the solution in Lemma 2.2, imply that

$$
\int_{0}^{1} \Phi\left(x, u\left(x, t_{n+1}\right)\right) d x \leq C_{1}
$$

with a constant $C_{1}$ that is independent of $\varepsilon, \mu$ and $n$. Hence, the equidistribution principle (1.3) leads to

$$
\alpha^{n+1} h_{m}^{n+1} \leq \int_{x_{m-1}^{n+1}}^{x_{m}^{n+1}} \Phi\left(x, u\left(x, t_{n+1}\right)\right) d x=\frac{1}{M} \int_{0}^{1} \Phi\left(x, u\left(x, t_{n+1}\right)\right) d x \leq C_{1} M^{-1},
$$

which implies that $h_{m}^{n+1} \leq C M^{-1}$, with a constant $C$ that is independent of $\varepsilon$ and $\mu$.

\section{Convergence Analysis}

In this section we derive the convergence analysis for the presented fully discrete scheme applied to the IBVP (1.1) by using the Lax Equivalence Theorem [10] and showing that the scheme is consistent and stable.

To obtain the error estimate for (2.8), consider first the difference equation

$$
\begin{aligned}
& \left(I+\Delta t L^{M}\right) \tilde{U}_{m}^{n+1}=r_{m, n+1}^{-} \tilde{U}_{m-1}^{n+1}+r_{m, n+1}^{*} \tilde{U}_{m}^{n+1}+r_{m, n+1}^{+} \tilde{U}_{m+1}^{n+1}=\tilde{g}_{m}^{n}, m=1, \cdots M-1, \\
& \tilde{U}_{0}^{n+1}=\tilde{U}_{M}^{n+1}=0,
\end{aligned}
$$

with $r_{m, n+1}^{-}, r_{m, n+1}^{+}$, and $r_{m, n+1}^{*}$ as in 2.12 , and $\tilde{g}_{m}^{n}:=u\left(x_{m}^{n+1}, t_{n}\right)+\Delta t f\left(x_{m}^{n+1}, t_{n+1}\right)$. We have the following lemma.

Lemma 3.1 If the difference operator $L^{M}$ defined in (2.9) satisfies the inequality

$$
\left(I+\Delta t L^{M}\right) Y_{m}^{n+1} \leq\left(I+\Delta t L^{M}\right) Z_{m}^{n+1}, 1 \leq m \leq M-1,
$$

with $Y_{0}^{n+1} \leq Z_{0}^{n+1}$ and $Y_{M}^{n+1} \leq Z_{M}^{n+1}$, then $Y_{m}^{n+1} \leq Z_{m}^{n+1}$ for $0 \leq m \leq M, 0 \leq n \leq N-1$. 
Proof. The stiffness matrix associated with the discrete operator $\left(I+\Delta t L^{M}\right)$ at every time level $t_{n+1}$ is an irreducibly diagonally dominant M-Matrix [26] and thus it has a nonnegative inverse. As a consequence, the assertion follows from the monotonicity of the inverse of $\left(I+\Delta t L^{M}\right)$.

Lemma 3.1 also shows that the difference equation (3.1) has a unique solution in every step.

For the error analysis, we again decompose the discrete solution $\tilde{U}_{m}^{n}$ of 3.1 into its regular component $\tilde{V}_{m}^{n}$ and singular component $\tilde{W}_{m}^{n}$ associated to the boundary layers, so that $\tilde{U}_{m}^{n}=\tilde{V}_{m}^{n}+\tilde{W}_{m}^{n}$, where

$$
\begin{aligned}
& \left(I+\Delta t L^{M}\right) \tilde{V}_{m}^{n+1}=v\left(x_{m}^{n+1}, t_{n}\right)+\Delta t f\left(x_{m}^{n+1}, t_{n+1}\right), 1 \leq m \leq M-1,0 \leq n \leq N-1, \\
& \tilde{V}_{0}^{n+1}=v\left(x_{0}^{n+1}, t_{n+1}\right), \tilde{V}_{M}^{n+1}=v\left(x_{M}^{n+1}, t_{n+1}\right),
\end{aligned}
$$

and

$$
\begin{aligned}
& \left(I+\Delta t L^{M}\right) \tilde{W}_{m}^{n+1}=w\left(x_{m}^{n+1}, t_{n}\right), 1 \leq m \leq M-1,0 \leq n \leq N-1, \\
& \tilde{W}_{0}^{n+1}=w\left(x_{0}^{n+1}, t_{n+1}\right), \tilde{W}_{M}^{n+1}=w\left(x_{M}^{n+1}, t_{n+1}\right) .
\end{aligned}
$$

Hence, the local error can be calculated by evaluating the error of the regular and singular component separately, and using the triangle inequality we obtain

$$
\left|\tilde{U}_{m}^{n+1}-\tilde{u}^{n+1}\left(x_{m}^{n+1}\right)\right| \leq\left|\tilde{V}_{m}^{n+1}-\tilde{v}^{n+1}\left(x_{m}^{n+1}\right)\right|+\left|\tilde{W}_{m}^{n+1}-\tilde{w}^{n+1}\left(x_{m}^{n+1}\right)\right| .
$$

In the following we denote the approximation error of the function $g$ at $x=x_{m}^{n}$ and $t=t_{n}$ by $\tau_{m}^{n}(g)$.

\subsection{The error of the regular component}

In this subsection we estimate the error of the solution in the regular component, i.e. outside the boundary layers. The following lemma gives an estimate for the local errors of $\tilde{V}_{m}^{n+1}$.

Lemma 3.2 The local error of the regular component $\tilde{V}_{m}^{n+1}$ satisfies

$$
\left|\tilde{V}_{m}^{n+1}-\tilde{v}^{n+1}\left(x_{m}^{n+1}\right)\right| \leq C M^{-1}, m=1, \cdots, M-1, n=0, \cdots, N-1 .
$$

Proof. By the Peano kernel theorem [1, the local error of $\tilde{V}_{m}^{n+1}$ at time $t=t_{n+1}$ can be determined from

$$
\begin{aligned}
\tau_{m}^{n+1}(\tilde{V})= & \left(I+\Delta t L^{M}\right)\left(\tilde{v}^{n+1}\left(x_{m}^{n+1}\right)-\tilde{V}_{m}^{n+1}\right) \\
= & -\frac{\varepsilon \Delta t}{h_{m}^{n+1}+h_{m+1}^{n+1}}\left\{\frac{1}{h_{m+1}^{n+1}} \int_{x_{m}^{n+1}}^{x_{m+1}^{n+1}}\left(s-x_{m+1}^{n+1}\right)^{2}\left(\frac{\partial^{3}}{\partial x^{3}} \tilde{v}^{n+1}\right)(s) d s\right. \\
& \left.-\frac{1}{h_{m}^{n+1}} \int_{x_{m-1}^{n+1}}^{x_{m}^{n+1}}\left(s-x_{m-1}^{n+1}\right)^{2}\left(\frac{\partial^{3}}{\partial x^{3}} \tilde{v}^{n+1}\right)(s) d s\right\} \\
& -\frac{\Delta t \mu b\left(x_{m}^{n+1}\right)}{h_{m}^{n+1}} \int_{x_{m}^{n+1}}^{x_{m+1}^{n+1}}\left(s-x_{m}^{n+1}\right)\left(\frac{\partial^{2}}{\partial x^{2}} \tilde{v}^{n+1}\right)(s) d s .
\end{aligned}
$$


Hence

$$
\left|\tau_{m}^{n+1}(\tilde{V})\right| \leq \varepsilon \Delta t \int_{x_{m-1}^{n+1}}^{x_{m+1}^{n+1}}\left|\frac{\partial^{3}}{\partial x^{3}} \tilde{v}^{n+1}(s)\right| d s+\beta_{1} \Delta t \mu \int_{x_{m}^{n+1}}^{x_{m+1}^{n+1}}\left|\frac{\partial^{2}}{\partial x^{2}} \tilde{v}^{n+1}(s)\right| d s \leq C M^{-1},
$$

where the derivative bounds from Lemma 2.2 and the step-size bounds from Lemma 2.5 are used. Now, since by Lemma 3.1 the discrete operator $\left(I+\Delta t L^{M}\right)$ is invertible with nonnegative inverse, we have

$$
\left|\tilde{V}_{m}^{n+1}-\tilde{v}^{n+1}\left(x_{m}^{n+1}\right)\right| \leq C M^{-1}, m=1, \cdots, M-1,
$$

at time $t=t_{n+1}$, where $n=0, \cdots, N-1$ and $C$ is independent of $\varepsilon, \mu$.

\subsection{The error in the singular component}

In the following two lemmas the local error in the singular component $\tilde{W}_{m}^{n+1}$ associated with the two boundary layers is analyzed.

Lemma 3.3 The singular component $\tilde{W}_{m}^{n+1}$ satisfies the error bound

$$
\left|\tilde{W}_{m}^{n+1}-\tilde{w}^{n+1}\left(x_{m}^{n+1}\right)\right| \leq C M^{-1}, m=k_{l}, \cdots, k_{r}, n=0, \cdots, N-1 .
$$

Proof. The analysis of the truncation error for the singular component $\tilde{W}_{m}^{n+1}$ yields

$$
\begin{aligned}
\left|\tau_{m}^{n+1}(\tilde{W})\right| & =\left|\left(I+\Delta t L^{M}\right)\left(\tilde{w}^{n+1}\left(x_{m}^{n+1}\right)-\tilde{W}_{m}^{n+1}\right)\right| \\
& =\left|r_{m, n+1}^{-} \tilde{w}^{n+1}\left(x_{m-1}^{n+1}\right)+r_{m, n+1}^{*} \tilde{w}^{n+1}\left(x_{m}^{n+1}\right)+r_{m, n+1}^{+} \tilde{w}^{n+1}\left(x_{m+1}^{n+1}\right)-\left(I+\Delta t L^{M}\right) \tilde{w}\left(x_{m}^{n+1}\right)\right| \\
& \leq C\left(\varepsilon\left\|\frac{\partial^{2}}{\partial x^{2}} \tilde{w}(x)\right\|_{\left[x_{m-1}^{n+1}, x_{m+1}^{n+1}\right]}+\mu\left\|\frac{\partial}{\partial x} \tilde{w}(x)\right\|_{\left[x_{m-1}^{n+1}, x_{m+1}^{n+1}\right]}\right) \\
& \leq C\left\{\begin{array}{l}
\exp \left(-p \Psi_{l} x_{m-1}^{n+1}\right), x_{m}^{n+1} \leq 1 / 2, \\
\exp \left(-p \Psi_{r}\left(1-x_{m+1}^{n+1}\right)\right), x_{m}^{n+1}>1 / 2,
\end{array}\right.
\end{aligned}
$$

where we have used our assumption that $\mu^{2} \leq \varepsilon$. Again, from 2.17 we have that

$$
\frac{\alpha^{n+1} x_{z_{l}-1}^{n+1}}{K}+K_{l}\left[1-\exp \left(-p \Psi_{l} \frac{x_{z_{l}-1}^{n+1}}{2}\right)\right]=\frac{2\left(z_{l}-1\right)}{M},
$$

which implies that

$$
\begin{aligned}
\exp \left(-p \Psi_{l} \frac{x_{z_{l}-1}^{n+1}}{2}\right) & \leq \frac{1}{K_{l}}\left[\frac{2}{p \Psi_{l}} \log (M)+K_{l}-\frac{1}{M}\left(K_{l}(M-1)+\frac{2 M}{p \Psi_{l}} \log (M)\right)+\frac{2}{M}\right] \\
& \leq C M^{-1},
\end{aligned}
$$

by using Lemma 2.3 . Therefore, for $z_{l} \leq m$ and $x_{m}^{n+1} \leq 1 / 2$, we have

$$
\left|\tau_{m, \tilde{W}}^{n+1}\right| \leq C \exp \left(-p \Psi_{l} x_{z_{l}-1}^{n+1}\right)=C\left(\exp \left(-p \Psi_{l} x_{z_{l}-1}^{n+1} / 2\right)\right)^{2} \leq C M^{-1}
$$

Hence, Lemma 3.1 implies that $\left|\tilde{W}_{m}^{n+1}-\tilde{w}^{n+1}\left(x_{m}^{n+1}\right)\right| \leq C M^{-1}$, where $C$ is independent of $\varepsilon, \mu, M$, and the mesh points $x_{M}^{n+1}$.

The error analysis for $z_{r} \geq m, x_{m}^{n+1}>1 / 2$ can be carried out analogously. 
Lemma 3.4 The singular component $\tilde{W}_{m}^{n+1}$ satisfies the following estimate.

$$
\left|\tilde{W}_{m}^{n+1}-\tilde{w}^{n+1}\left(x_{m}^{n+1}\right)\right| \leq C M^{-1}, m=1, \cdots, z_{l}-1, z_{r}+1, \cdots, M-1 .
$$

Proof. We only consider the left boundary layer, the proof for the right boundary layer is analogous. A direct calculation using the Taylor series expansion shows that

$$
\begin{gathered}
\tau_{m, \tilde{W}}^{n+1}=\frac{\varepsilon\left(h_{m}^{n+1}\right)^{2}\left(\frac{\partial^{3}}{\partial x^{3}} \tilde{w}^{n+1}\right)\left(\xi_{m}^{1}\right)}{3\left(h_{m}^{n+1}+h_{m+1}^{n+1}\right)}-\frac{\varepsilon\left(h_{m+1}^{n+1}\right)^{2}\left(\frac{\partial^{3}}{\partial x^{3}} \tilde{w}^{n+1}\right)\left(\xi_{m}^{2}\right)}{3\left(h_{m}^{n+1}+h_{m+1}^{n+1}\right)} \\
+\frac{\mu}{h_{m+1}^{n+1}} \int_{x_{m}^{n+1}}^{x_{m+1}^{n+1}}\left(s-x_{m}^{n+1}\right)\left(\frac{\partial^{2}}{\partial x^{2}} \tilde{w}^{n+1}(s)\right) d s .
\end{gathered}
$$

Hence

$$
\begin{aligned}
\left|\tau_{m, \tilde{W}}^{n+1}\right| \leq C \Psi_{l}^{2} & \int_{x_{m}^{n+1}}^{x_{m+1}^{n+1}} \exp \left(-p \Psi_{l} x\right) d x \\
& +C \mu \int_{x_{m}^{n+1}}^{x_{m+1}^{n+1}}\left|\left(\frac{\partial^{2}}{\partial x^{2}} \tilde{w}^{n+1}\right)\right| d s
\end{aligned}
$$

where $\xi_{m}^{1} \in\left(x_{m-1}^{n+1}, x_{m}^{n+1}\right)$ and $\xi_{m}^{2} \in\left(x_{m}^{n+1}, x_{m+1}^{n+1}\right)$. Setting $\Lambda_{\Phi}=\int_{0}^{1} \Phi\left(x, u\left(x, t^{n+1}\right)\right) d x$, then we obtain

$$
\begin{aligned}
\mathcal{I}_{1}:= & C \Psi_{l}^{2} \int_{x_{m-1}^{n+1}}^{x_{m+1}^{n+1}} \exp \left(-p \Psi_{l} x\right) d x \\
& \leq C \Psi_{l}^{2} \int_{\xi_{m-1}^{n+1}}^{\xi_{m+1}^{n+1}} \frac{\exp \left(-p \Psi_{l} x(\xi)\right) \Lambda_{\Phi}}{\Psi(x(\xi))} d \xi=C \Psi_{l}^{2} \int_{\xi_{m-1}^{n+1}}^{\xi_{m+1}^{n+1}} \frac{\exp \left(-p \Psi_{l} x(\xi)\right) \Lambda_{\Phi}}{\alpha_{n+1}+\left|\tilde{w}_{\xi \xi}\right|^{1 / 2}} d \xi \\
& \leq C \Psi_{l} \int_{\xi_{m-1}^{n+1}}^{\xi_{m+1}^{n+1}} \exp \left(-p \Psi_{l} x(\xi) / 2\right) d \xi \leq C M^{-1} \Psi_{l} \exp \left(-p \Psi_{l} x_{m-1}^{n+1} / 2\right)\left(\xi_{m+1}^{n+1}-\xi_{m-1}^{n+1}\right) \\
& \leq C M^{-1} \Psi_{l} \exp \left(-p \Psi_{l} x_{m-1}^{n+1} / 2\right) \\
& =C M^{-1} \Psi_{l} \exp \left(-p \Psi_{l} x_{m}^{n+1} / 2\right) \exp \left(p \Psi_{l} h_{m}^{n+1} / 2\right) .
\end{aligned}
$$

By Lemma 2.4 we have that $\Psi_{l} h_{m}^{n+1} \leq C$ for $m=1, \cdots, z_{l}-1$, and hence, $\exp \left(p \Psi_{l} h_{m}^{n+1} / 2\right)<$ $C$, which implies that $\mathcal{I}_{1} \leq C \Psi_{l} M^{-1} \exp \left(-p \Psi_{l} x_{m}^{n+1} / 2\right)$. An analogous calculation shows that

$$
\mathcal{I}_{2}:=\int_{x_{m}^{n+1}}^{x_{m+1}^{n+1}}\left(s-x_{m}^{n+1}\right)\left(\frac{\partial^{2}}{\partial x^{2}} \tilde{w}^{n+1}(s)\right) d s \leq C \Psi_{l} M^{-1} \exp \left(-p \Psi_{l} x_{m}^{n+1} / 2\right) .
$$

Again

$\exp \left(-p \Psi_{l} x_{m}^{n+1} / 2\right)=\exp \left(\sum_{k=1}^{m}-p \Psi_{l} h_{k}^{n+1} / 2\right)=\prod_{k=1}^{m} \exp \left(-p \Psi_{l} h_{k}^{n+1} / 2\right)<\prod_{k=1}^{m}\left(1+p \Psi_{l} h_{k}^{n+1} / 2\right)^{-1}$,

since $\exp (-\gamma)<1 /(1+\gamma)$ for any positive real number $\gamma$. Set $S_{m}^{n+1}:=\prod_{k=1}^{m}\left(1+p \Psi_{l} h_{k}^{n+1} / 2\right)^{-1}$ with $S_{0}^{n+1}=1$, so that $\mathcal{I}_{j} \leq C \Psi_{l} M^{-1} S_{m}^{n+1}$ for $j=1,2$. 
A straight calculation shows that

$$
\begin{aligned}
(I+ & \left.\Delta t L^{N}\right) S_{m}^{n+1}=r_{m, n+1}^{-} S_{m-1}^{n+1}+r_{m, n+1}^{c} S_{m}^{n+1}+r_{m, n+1}^{+} S_{m+1}^{n+1} \\
& =S_{m}^{n+1} \frac{\Psi_{l} p}{2+p \Psi_{l} h_{m+1}^{n+1}}\left[-\frac{p \varepsilon \Psi_{l} h_{m+1}^{n+1}}{h_{m}^{n+1}+h_{m+1}^{n+1}}+\mu b_{m}^{n+1}\right]+\left(1+c_{m}^{n+1}\right) S_{m}^{n+1} \\
& \geq S_{m}^{n+1} \frac{\Psi_{l} p}{2+p \Psi_{l} h_{m+1}^{n+1}}\left[\mu\left(b_{m}^{n+1}-\frac{p b_{m}^{n+1} h_{m+1}^{n+1}}{h_{m}^{n+1}+h_{m+1}^{n+1}}\right)-\frac{p h_{m+1}^{n+1} \sqrt{\varepsilon c_{m}^{n+1}}}{h_{m}^{n+1}+h_{m+1}^{n+1}}+\right. \\
& \left.+c_{m}^{n+1} \frac{2+p \Psi_{l} h_{m+1}^{n+1}}{p \Psi_{l}}\right]+S_{m}^{n+1} \\
& =S_{m}^{n+1} \frac{\Psi_{l} p}{2+p \Psi_{l} h_{m+1}^{n+1}}\left[\mu A_{1}+A_{2}\right]+S_{m}^{n+1}
\end{aligned}
$$

where $A_{1}>0$ and

$$
\begin{aligned}
A_{2} & =c_{m}^{n+1} \frac{2+p \Psi_{l} h_{m+1}^{n+1}}{p \Psi_{l}}-\frac{p h_{m+1}^{n+1} \sqrt{\varepsilon c_{m}^{n+1}}}{h_{m}^{n+1}+h_{m+1}^{n+1}} \\
& >-p c_{m}^{n+1} \sqrt{\varepsilon}+\frac{p h_{m}^{n+1} \sqrt{\varepsilon c_{m}^{n+1}}}{h_{m}^{n+1}+h_{m+1}^{n+1}}+c_{m}^{n+1}\left[\frac{2+p \Psi_{l} h_{m+1}^{n+1}}{p \Psi_{l}}\right]>0,
\end{aligned}
$$

since $c(t, x)>1$ and $2 \geq p^{2} \Psi_{l} \sqrt{\varepsilon}$ for sufficiently small $\varepsilon$ and $\mu$. Therefore, we have

$$
\left(I+\Delta t L^{M}\right) S_{m}^{n+1} \geq C \frac{S_{m}^{n+1} p \Psi_{l}}{2+p \Psi_{l} h_{m+1}^{n+1}}\left[A_{1}+A_{2}\right] \geq \frac{C}{\max \left(2 / p \Psi_{l}, h_{m+1}^{n+1}\right)} S_{m}^{n+1} .
$$

With the function $\lambda_{m}^{n+1}=C M^{-1}\left(1+S_{m}^{n+1}\right)$, for $m=0, \cdots, z_{l}-1, z_{r}+1, \cdots, M$, we have

$$
\begin{aligned}
\left(I+\Delta t L^{M}\right)\left(\tilde{w}^{n+1}\left(x_{m}^{n+1}\right)-\tilde{W}_{m}^{n+1}\right) & \leq C \Psi_{l} M^{-1} S_{m}^{n+1} \\
& \leq C M^{-1}\left(I+\Delta t L^{M}\right) S_{m}^{n+1} \\
& \leq\left(I+\Delta t L^{M}\right) \lambda_{m}^{n+1}
\end{aligned}
$$

with $\left|\tilde{w}^{n+1}\left(x_{m}^{n+1}\right)-\tilde{W}_{m}^{n+1}\right|=0<\lambda_{m}^{n+1}$ for $m=0, M$, and $\left|\tilde{w}^{n+1}\left(x_{m}^{n+1}\right)-\tilde{W}_{m}^{n+1}\right|<C M^{-1}<$ $\lambda_{m}^{n+1}$ for $m=z_{l}-1, z_{r}+1$. Therefore, Lemma 3.1 implies that

$$
\left|\tilde{W}_{m}^{n+1}-\tilde{w}^{n+1}\left(x_{m}^{n+1}\right)\right| \leq C M^{-1}, \quad m=1, \cdots, z_{l}-1, z_{r}+1, \cdots, M-1,
$$

which completes the proof.

Theorem 3.5 Let $\tilde{u}^{n+1}$ and $\tilde{U}^{n+1}$ be the solutions of 2.5) and (3.1), respectively. Then, at each time step the following estimate holds

$$
\left|\tilde{U}_{m}^{n+1}-\tilde{u}^{n+1}\left(x_{m}^{n+1}\right)\right| \leq C M^{-1}, m=1, \cdots, M,
$$

where $C$ is independent of $\varepsilon, \mu, M$ and the mesh points $x_{m}^{n+1}$. 
Proof. The assertion follows from Lemmas 3.23 .4 and the inequality

$$
\left|\tilde{U}_{m}^{n+1}-\tilde{u}^{n+1}\left(x_{m}^{n+1}\right)\right| \leq\left|\tilde{V}_{m}^{n+1}-\tilde{v}^{n+1}\left(x_{m}^{n+1}\right)\right|+\left|\tilde{W}_{m}^{n+1}-\tilde{w}^{n+1}\left(x_{m}^{n+1}\right)\right| .
$$

As a corollary we have the following estimate.

Corollary 3.6 Let $u\left(x, t_{n+1}\right)$ and $U^{n+1}$ be the solution of (1.1) and its fully discretized problem (2.8), respectively, in $\Omega_{x}^{n+1}$ and in the $(n+1)$ st time step $t_{n+1}=(n+1) \Delta t$. If we assume that $M^{-q} \leq C \Delta t$ with $0<q<1$, then

$$
\left|\tilde{U}_{m}^{n+1}-\tilde{u}^{n+1}\left(x_{m}^{n+1}\right)\right| \leq C \Delta t M^{-1+q}, m=1, \cdots, m-1 .
$$

With the help of Corollary 3.6, we can prove the main theorem of this paper, which shows that the solution of the fully discrete scheme (2.8) converges to the solution $u(x, t)$ on the final layer adapted mesh obtained by the equidistribution via the monitor function 2.14).

Theorem 3.7 Let $u\left(x, t_{n+1}\right)$ and $\left\{U^{n+1}\right\}$ be the solution of the IBVP (1.1) and its fully discretized problem (2.8), respectively, in $\Omega_{x}^{n+1}$ at the $(n+1)$ st time step $t_{n+1}=(n+1) \Delta t$. If we assume that $M^{-q} \leq C \Delta t$ with $0<q<1$, then the error of the fully discrete scheme in each time step $t_{n+1}$ satisfies

$$
\left\|U_{m}^{n+1}-u\left(x_{m}^{n+1}, t_{n+1}\right)\right\|_{\mathcal{I}} \leq C\left(M^{-1+q}+\Delta t\right), x_{m}^{n+1} \in \Omega_{x}^{n+1},
$$

where $\mathcal{I}$ is the index set $\{0,1, \cdots, M\},\|\|_{\mathcal{I}}$ denotes the maximum norm over all the indices from $\mathcal{I}$ and $C$ is independent of $\varepsilon, \mu, M$ and the mesh points $x_{m}^{n+1}$.

Proof. At time step $t_{n}$, let us define the error of the fully discrete scheme as $E_{m}^{n}=$ $U_{m}^{n}-u\left(x_{m}^{n}, t_{n}\right)$, where $m=1, \cdots, M$. Then, using (3.4) and Lemma 2.1 we obtain

$$
\begin{aligned}
\left\|E_{m}^{n}\right\|_{\mathcal{I}} & \leq\left\|U_{m}^{n}-\tilde{U}_{m}^{n}\right\|_{\mathcal{I}}+\left\|\tilde{U}_{m}^{n}-\tilde{u}_{m}^{n}\right\|_{\mathcal{I}}+\left\|\tilde{u}_{m}^{n}-u\left(x_{m}^{n}, t_{n}\right)\right\|_{\mathcal{I}} \\
& \leq\left\|U_{m}^{n}-\tilde{U}_{m}^{n}\right\|_{\mathcal{I}}+C \Delta t\left(M^{-1+q}+\Delta t\right),
\end{aligned}
$$

The solution of the fully discrete problem is stable. Therefore, $\left\|U_{m}^{n}-\tilde{U}_{m}^{n}\right\|_{I} \leq C|| \bar{U}\left(x_{m}^{n}, t_{n-1}\right)-$ $u\left(x_{m}^{n}, t_{n-1}\right) \|_{\mathcal{I}}$, and, by setting $\delta_{k}(x)=\left(x-x_{k-1}^{n-1}\right) /\left(x_{k}^{n-1}-x_{k-1}^{n-1}\right), \delta_{k-1}(x)=1-\delta_{k}(x)$ where $x_{k-1}^{n-1} \leq x_{m}^{n} \leq x_{k}^{n-1}$ for some $k$, we have

$$
\begin{aligned}
& \left\|(u-\bar{U})\left(x_{m}^{n}, t_{n-1}\right)\right\|_{\mathcal{I}} \\
& =\left\|u\left(x_{m}^{n}, t_{n-1}\right)-\left(U\left(x_{k-1}^{n-1}, t_{n-1}\right) \delta_{k-1}\left(x_{m}^{n}\right)+U\left(x_{k}^{n-1}, t_{n-1}\right) \delta_{k}\left(x_{m}^{n}\right)\right)\right\|_{\mathcal{I}} \\
& \leq\left\|u\left(x_{m}^{n}, t_{n-1}\right)-\left(u\left(x_{k-1}^{n-1}, t_{n-1}\right) \delta_{k-1}\left(x_{m}^{n}\right)+u\left(x_{k}^{n-1}, t_{n-1}\right) \delta_{k}\left(x_{m}^{n}\right)\right)\right\|_{\mathcal{I}} \\
& +\left\|(u-U)\left(x_{k-1}^{n-1}, t_{n-1}\right) \delta_{k-1}\left(x_{m}^{n}\right)+(u-U)\left(x_{k}^{n-1}, t_{n-1}\right) \delta_{k}\left(x_{m}^{n}\right)\right\|_{\mathcal{I}} \\
& \leq\left\|u\left(x_{m}^{n}, t_{n-1}\right)-\left(u\left(x_{k-1}^{n-1}, t_{n-1}\right) \delta_{k-1}\left(x_{m}^{n}\right)+u\left(x_{k}^{n-1}, t_{n-1}\right) \delta_{k}\left(x_{m}^{n}\right)\right)\right\|_{\mathcal{I}}+\left\|E_{m}^{n-1}\right\|_{\mathcal{I}},
\end{aligned}
$$

Now, it is shown in 2 that the interpolation error is bounded by $M^{-1}$, which implies $\left\|u\left(x_{m}^{n}, t_{n-1}\right)-\left(u\left(x_{k-1}^{n-1}, t_{n-1}\right) \delta_{k-1}\left(x_{m}^{n}\right)+u\left(x_{k}^{n-1}, t_{n-1}\right) \delta_{k}\left(x_{m}^{n}\right)\right)\right\|_{\mathcal{I}} \leq C \Delta t M^{-1+q}$. Therefore, we have the following recurrence relation

$$
\left\|E_{m}^{n}\right\|_{\mathcal{I}} \leq\left\|E_{m}^{n-1}\right\|+C \Delta t\left(M^{-1+q}+\Delta t\right), \quad \text { for } \quad m \in \mathcal{I},
$$

from which the required result follows.

Remark 3.8 The described adaptive method and also the error estimates can also be used to solve (1.1) in the case that $\varepsilon=0$. 


\section{Numerical Experiments}

In this section we present several numerical experiments to illustrate the theoretical results, derived in the previous section. The generation of the adaptive finite difference solution requires two steps. First the equidistributed mesh and the corresponding solution have to be determined by a mesh generation algorithm at each time step, and then the final mesh and the final solution will be calculated using the solution at previous time steps.

\subsection{Adaptive mesh generation algorithm}

We use the following iterative algorithm to generate the layer adapted mesh by the equidistribution of the monitor function (2.14). A similar algorithm was suggested for steady state convection-diffusion type problems in [5, 17]. The convergence of this algorithm is addressed in [29]. Here, our aim is to construct a mesh that solves the following equidistribution problem

$$
\Phi_{m}^{n} h_{m}^{n}=\frac{1}{N} \sum_{j=1}^{N} \Phi_{j}^{n} h_{j}^{n}, m=1, \cdots, M,
$$

where $\Phi_{m}^{n}$ is the discrete approximation of the monitor function $\Phi\left(x, u\left(x, t_{n}\right)\right)$ in the subinterval $\left(x_{m-1}^{n}, x_{m}^{n}\right)$. Observe that instead of solving the discretized equidistribution problem 1.3. exactly, it is enough to stop the algorithm when

$$
\Phi_{m}^{n} h_{m}^{n} \leq \frac{C_{0}}{M} \sum_{j=1}^{M} \Phi_{j}^{n} h_{j}^{n}, m=1, \cdots, M,
$$

where $C_{0}>1$ is a user defined constant. Choosing $C_{0}$ larger will lead to fewer iterations

for the algorithm. If, however, $C_{0}$ is close to 1 , then this leads to a more accurate solution obtained with many iterations.

\section{Algorithm 1}

1. Start with $n=1$.

2. Define the uniform mesh $\left\{x^{n,(0)}: x_{m}^{n,(0)}=m / N, 0 \leq m \leq M\right\}$ as initial mesh for $n=1$, otherwise define $\left\{x^{n-1}\right\}$ as the initial mesh at the $n$th time step and go to Step 3. assuming $p=0$.

3. Solve the discrete problem 2.8 on the mesh $\left\{x^{n,(p)}\right\}$ for $\left\{U^{n,(p)}\right\}$ and 2.13 for $\left\{V^{n,(p)}\right\}$. Now define $h_{m}^{n,(p)}=x_{m}^{n,(p)}-x_{m-1}^{n,(p)}$ for $m=1, \cdots, M$.

4. Denote the singular component of the discrete solution $U_{m}^{n,(p)}$ as $W_{m}^{n,(p)}=U_{m}^{n,(p)}-V_{m}^{n,(p)}$, for $m=1, \cdots, M$. Determine the discretized monitor function

$$
\Phi_{m}^{n,(p)}=\alpha^{n,(p)}+\left|\bar{\Delta}_{x}^{2} W_{m}^{n,(p)}\right|^{1 / 2} m=1, \cdots, M .
$$

by defining $\bar{\Delta}_{x}^{2} W_{m}^{n,(p)}=\left(\Delta_{x}^{2} W_{m}^{n,(p)}+\Delta_{x}^{2} W_{m-1}^{n,(p)}\right) / 2$ with $\bar{\Delta}_{x}^{2} W_{m}^{n,(p)}=\Delta_{x}^{2} W_{1}^{n,(p)}$ and $\bar{\Delta}_{x}^{2} W_{M}^{n,(p)}=\Delta_{x}^{2} W_{M-1}^{n,(p)}$, where $\alpha^{n,(p)}=\sum_{m=1}^{M} h_{m}^{n,(p)}\left|\bar{\Delta}_{x}^{2} W_{m}^{n,(p)}\right|^{1 / 2}$.

Compute

$$
\Gamma_{j}^{n,(p)}=\sum_{m=1}^{j} h_{m}^{n,(p)} \Phi_{m}^{n,(p)} .
$$


5. Choose a constant $C_{0}>1$. If the stopping criteria for the iteration

$$
\frac{\max _{m=1, \ldots, M} h_{m}^{n,(p)} \Phi_{m}^{n,(p)}}{\Gamma_{M}^{n,(p)}} \leq \frac{C_{0}}{M} .
$$

is satisfied, then go to Step 7., else continue with Step 6.

6. Generate a new mesh by equidistributing the proposed monitor function using the current computed solution from Step 3. and $\Gamma_{m}^{n,(p)}$ from Step 4. Set $Y_{m}^{n,(p)}=m \Gamma_{M}^{n,(p)} / M$ for $m=0, \cdots, M$. Now interpolate $\left(Y_{m}^{n,(p)}, x_{m}^{n,(p+1)}\right)$ to $\left(\Gamma_{m}^{n,(p)}, x_{m}^{n,(p)}\right)$ using piecewise linear interpolation. Generate a new mesh $x^{n,(p+1)} \equiv\left\{0=x_{0}^{n,(p+1)}<x_{1}^{n,(p+1)}<\cdots<\right.$ $\left.x_{M}^{n,(p+1)}=1\right\}$ and return to Step 3.

7. Set $x^{n, *}=\left\{0=x_{0}^{n, *}<x_{1}^{n, *}<\cdots<x_{M}^{n, *}=1\right\}=x^{n,(p+1)}$ and $U^{n, *}=U^{n,(p+1)}$, where $U^{n, *}$ is the layer adapted solution at $n$th time step.

8. If $n=N$, then $U^{n, *}$ is the solution on the adapted mesh $x^{n, *}$ at the final time. Otherwise, set $n=n+1$, go to Step 2., and repeat the process to find the required solution $U^{n, *}$ at $x^{n, *}$.

\section{$4.2 \quad$ Numerical examples}

To illustrate the accuracy of the method and the theoretical results of error analysis, we present two numerical examples, where the meshes are equidistributed by the monitor function 2.14. For these two test problems, the errors and the corresponding rates of convergence are displayed in several tables.

Example 4.1 Our first example is the parabolic IBVP

$$
\begin{aligned}
& u_{t}-\varepsilon u_{x x}+\mu(1+\exp (x)) u_{x}+\left(1+x^{4}|x|\right) u=10 \exp \left(t^{2}\right)\left(x^{2}-x^{4}\right), \quad(x, t) \in(0,1) \times(0,1], \\
& u(x, 0)=x^{3}(1-x)^{3}, \quad x \in(0,1), \\
& u(0, t)=u(1, t)=0, \quad t \in(0,1] .
\end{aligned}
$$

Example 4.2 The second example is the IBVP for $(x, t) \in(0,1) \times(0,1]$ given by

$$
\begin{aligned}
& u_{t}-\varepsilon u_{x x}+\mu\left(1+x(1-x)+t^{2}\right) u_{x}+(1+5 x t) u=x(x-1)(\exp (t)-1), \\
& u(x, 0)=0, \quad x \in(0,1) \\
& u(0, t)=u(1, t)=0, \quad t \in(0,1] .
\end{aligned}
$$

Since the exact solution of Examples 4.1 and 4.2 are not known, the accuracy of the numerical solutions will be determined by using a twice refined mesh, which for the computed mesh $\Omega_{x}^{M} \times \Omega_{t}^{N}$ uses the mesh $\Omega_{x}^{2 M} \times \Omega_{t}^{2 N}$ with $2 M$ spacial and $2 N$ time intervals. by

For any fixed value of $M$, the maximum error $E_{\varepsilon, \mu}^{M, \Delta t}$ over all time steps will be calculated

$$
E_{\varepsilon, \mu}^{M, \Delta t}=\max _{x_{m}^{n} \in \Omega_{x}^{M}, t_{n} \in \Omega_{t}^{N}}\left|U_{\varepsilon, \mu}^{M, \Delta t}\left(x_{m}^{n}, t_{n}\right)-U_{\varepsilon, \mu}^{2 M, \Delta t / 2}\left(x_{m}^{n}, t_{n}\right)\right|
$$




\begin{tabular}{cccccc}
\hline$\varepsilon, \mu$ & \multicolumn{5}{c}{ Number of space intervals $M /$ time step size $\Delta t$} \\
& $64 / \frac{1}{16}$ & $128 / \frac{1}{32}$ & $256 / \frac{1}{64}$ & $512 / \frac{1}{128}$ & $1024 / \frac{1}{256}$ \\
\hline$E_{10^{-2}, 10^{-4}}^{M, \Delta t}$ & 0.022542 & 0.011005 & 0.005392 & 0.002687 & 0.001337 \\
$r_{10^{-2}, 10^{-4}}^{M, \Delta t}$ & 1.03446 & 1.029256 & 1.00484 & 1.00658 & - \\
\hline$E_{10^{-3}, 10^{-4}}^{M,}$ & 0.024729 & 0.011976 & 0.005910 & 0.002947 & 0.001469 \\
$r_{10^{-3}, 10^{-4}}^{M, \Delta t}$ & 1.04599 & 1.01877 & 1.00397 & 1.00358 & - \\
\hline$E_{10^{-4}, 10^{-4}}^{M, \Delta t}$ & 0.024125 & 0.011985 & 0.005916 & 0.002966 & 0.001478 \\
$r_{10^{-4}, 10^{-4}}^{M, \Delta t}$ & 1.00925 & 1.01833 & 0.99607 & 1.00508 & - \\
\hline$E_{10^{-5}, 10^{-4}}^{M, \Delta t}$ & 0.024858 & 0.0120106 & 0.005923 & 0.002968 & 0.001485 \\
$r_{10^{-5}, 10^{-4}}^{M, \Delta t}$ & 1.04940 & 1.01984 & 0.99673 & 0.99913 & - \\
\hline Max. No. Iter. & 6 & 7 & 10 & 19 & 35 \\
\hline
\end{tabular}

Table 1: Maximum errors and orders of convergence for Example 4.1 over all time levels.

where $U_{\varepsilon, \mu}^{M, \Delta t}\left(x_{m}^{n}, t_{n}\right)$ is the computed solution with $M$ space and $N$ time intervals and $U_{\varepsilon, \mu}^{2 M, \Delta t / 2}\left(x_{m}^{n}, t_{n}\right)$ is the numerical solution at $\left(x_{m}^{n}, t_{n}\right)$ with $2 M$ space and $2 N$ time intervals.

The corresponding order of convergence is calculated by the formula

$$
r_{\varepsilon, \mu}^{M, \Delta t}=\log _{2}\left(\frac{E_{\varepsilon, \mu}^{M, \Delta t}}{E_{\varepsilon, \mu}^{2 M, \Delta t / 2}}\right),
$$

and the uniform error $E^{M}$ and corresponding order of convergence $r^{M}$ over a large set of parameters $\mathcal{P}=\left\{(\varepsilon, \mu) \mid \mu^{2} \leq \varepsilon, \varepsilon=1,10^{-1}, \cdots, 10^{-10}, \mu=1,10^{-1}, \cdots, 10^{-10}\right\}$ and all time steps as

$$
E^{M}=\max _{(\varepsilon, \mu) \in \mathcal{P}} E_{\varepsilon, \mu}^{M, \Delta t}, r^{M}=\log _{2}\left(\frac{E^{M}}{E^{2 M}}\right)
$$

respectively.

We also compare the uniform error (let us denote it by $E_{E q u i}^{M}$ ) obtained by the mesh equidistribution technique with the error $E_{\text {Shish }}^{M}$ obtained on a Shishkin mesh (see [24]) over all time steps. The Shishkin mesh is defined as a piecewise uniform mesh in the intervals $[0, \tau],[\tau, 1-\tau]$ and $[1-\tau, 1]$, where $\tau=\min (1 / 4,2 \sqrt{(\varepsilon / \sigma)} \ln (M))$. The $\sigma$ is considered as $2 /(1+e)$ and $4 / 5$ for Examples 4.1 and 4.2 .

For Example 4.1. Tables 1 and 2 and for Example 4.2 Tables 4 and 5 display the maximum $\varepsilon$-uniform errors and the corresponding orders of convergence for the numerical solution computed with our proposed monitor function using $C_{0}=2$ in the algorithm. The results show that the numerical solution is first-order uniformly accurate. The monotonically decreasing behavior of the errors can be observed from these tables as $M$ increases. These tables also show the maximum number of iterations for both examples over all time levels which indicates the convergence of the algorithm. Tables 3 and 7 give a comparison of parameter 


\begin{tabular}{|c|c|c|c|c|c|}
\hline \multirow[t]{2}{*}{$\varepsilon, \mu$} & \multicolumn{5}{|c|}{ Number of space intervals $M /$ time step size $\Delta t$} \\
\hline & $64 / \frac{1}{16}$ & $128 / \frac{1}{32}$ & $256 / \frac{1}{64}$ & $512 / \frac{1}{128}$ & $1024 / \frac{1}{256}$ \\
\hline$E_{10^{-5}, 10^{-6}}^{M, \Delta t}$ & 0.024987 & 0.011856 & 0.005886 & 0.002974 & 0.001484 \\
\hline$r_{10^{-5}, 10^{-6}}^{M, \Delta t}$ & 1.07552 & 1.01013 & 0.98506 & 1.00238 & - \\
\hline$E_{10^{-6}, 10^{-6}}^{M, \Delta t}$ & 0.025226 & 0.011922 & 0.005909 & 0.002961 & 0.001479 \\
\hline$r_{10^{-6}, 10^{-6}}^{M, \Delta t^{2}}$ & 1.08133 & 1.01249 & 0.99672 & 1.00169 & - \\
\hline$E_{10^{-7}, 10^{-6}}^{M, \Delta t}$ & 0.024718 & 0.012279 & 0.005906 & 0.002961 & 0.001472 \\
\hline$r_{10^{-7}, 10^{-6}}^{M, \Delta t}$ & 1.00931 & 1.05596 & 0.99620 & 1.00783 & - \\
\hline$E_{10^{-8}, 10^{-6}}^{M, \Delta t}$ & 0.024596 & 0.012064 & 0.006008 & 0.002981 & 0.001496 \\
\hline$r_{10^{-8}, 10^{-6}}^{M, \Delta t}$ & 1.02765 & 1.00568 & 1.01104 & 0.99503 & - \\
\hline Max. No. Iter. & 8 & 23 & 28 & 37 & 55 \\
\hline
\end{tabular}

Table 2: Maximum errors and orders of convergence for Example 4.1 over all time levels.

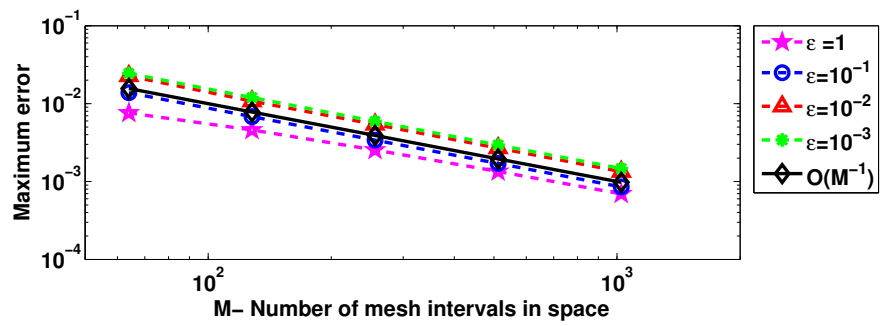

Figure 1: Log-log error plot at $t=1$ with $\mu=10^{-5}$ for Example 4.1 .

\begin{tabular}{cccccc}
\hline$(\varepsilon, \mu) \in \mathcal{P}$ & \multicolumn{6}{c}{ Number of space intervals $M /$ time step size $\Delta t$} \\
& $64 / \frac{1}{16}$ & $128 / \frac{1}{32}$ & $256 / \frac{1}{64}$ & $512 / \frac{1}{128}$ & $1024 / \frac{1}{256}$ \\
\hline$E_{E q u i}^{M}$ & 0.026044 & 0.013120 & 0.006214 & 0.003130 & 0.001555 \\
$r_{E q u i}^{M}$ & 0.98920 & 1.07808 & 0.98963 & 1.00889 & - \\
\hline$E_{S h i s h}^{M}$ & 0.025664 & 0.012675 & 0.008537 & 0.005505 & 0.003377 \\
$r_{\text {Shish }}^{M}$ & 1.017749 & 0.57018 & 0.63305 & 0.70457 & - \\
\hline
\end{tabular}

Table 3: Uniform errors and orders of convergence for Example 4.1 over all time steps. 


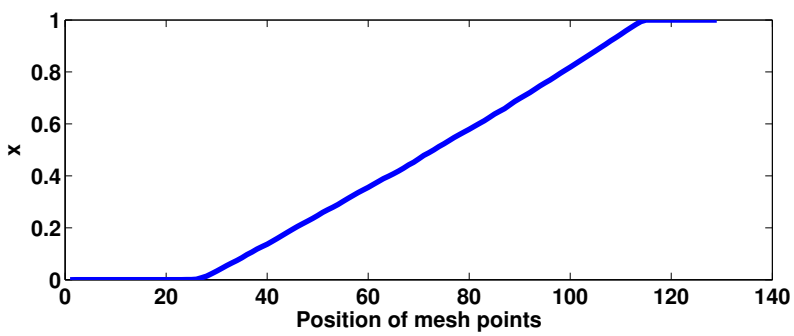

Figure 2: Mesh density towards boundary layers for $M=128$ at $t=1$ for Example 4.1 .

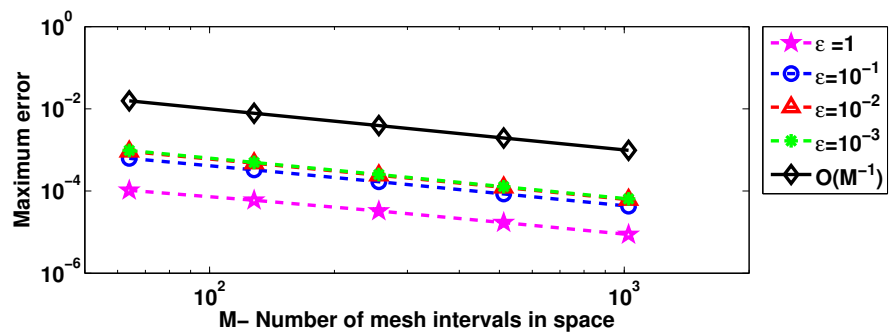

Figure 3: Log-log plot error at time $t=1$ with $\mu=10^{-5}$ for Example 4.2 . 


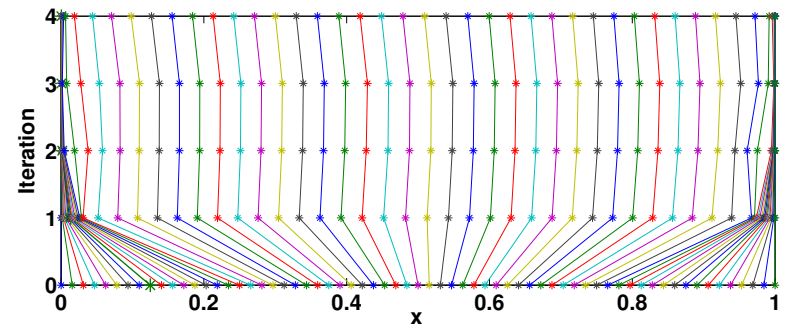

Figure 4: Mesh trajectory at $\varepsilon=10^{-6}, \mu=10^{-7}$ with $M=64$ for Example 4.2 at $t=1$.

\begin{tabular}{cccccc}
\hline$\varepsilon, \mu$ & \multicolumn{5}{c}{ Number of space intervals $M /$ time step size $\Delta t$} \\
& $64 / \frac{1}{16}$ & $128 / \frac{1}{32}$ & $256 / \frac{1}{64}$ & $512 / \frac{1}{128}$ & $1024 / \frac{1}{256}$ \\
\hline$E_{1,10^{-4}}^{M, \Delta t}$ & $0.010401 \mathrm{e}-2$ & $0.005949 \mathrm{e}-2$ & $0.003255 \mathrm{e}-2$ & $0.001705 \mathrm{e}-2$ & $0.000873 \mathrm{e}-2$ \\
$r_{1,10^{-4}}^{M,-2}$ & 0.80603 & 0.87015 & 0.93236 & 0.96576 & - \\
\hline$E_{10^{-1}, 10^{-4}}^{M, \Delta t}$ & $0.062215 \mathrm{e}-2$ & $0.032707-2$ & $0.016778 \mathrm{e}-2$ & $0.008504 \mathrm{e}-2$ & $0.004274 \mathrm{e}-2$ \\
$r_{10^{-1}, 10^{-4}}^{M,}$ & 0.92766 & 0.96307 & 0.98040 & 0.99245 & - \\
\hline$E_{10^{-2}, 10^{-4}}^{M, \Delta t}$ & $0.092026 \mathrm{e}-2$ & $0.047554 \mathrm{e}-2$ & $0.024197 \mathrm{e}-2$ & $0.012218 \mathrm{e}-2$ & $0.006141 \mathrm{e}-2$ \\
$r_{10^{-2}, 10^{-4}}^{M, \Delta t}$ & 0.95247 & 0.97475 & 0.98578 & 0.99238 & - \\
\hline$E_{10^{-3}, 10^{-4}}^{M, \Delta t}$ & $0.097148 \mathrm{e}-2$ & $0.049478 \mathrm{e}-2$ & $0.025256 \mathrm{e}-2$ & $0.012766 \mathrm{e}-2$ & $0.006411 \mathrm{e}-2$ \\
$r_{10^{-3}, 10^{-4}}^{M}$ & 0.97341 & 0.97014 & 0.98434 & 0.99355 & - \\
\hline Max. No. Iter. & 3 & 5 & 11 & 16 & 26 \\
\hline
\end{tabular}

Table 4: Maximum errors and orders of convergence for Example 4.2 over all time levels.

\begin{tabular}{cccccc}
\hline$\varepsilon, \mu$ & \multicolumn{5}{c}{ Number of space intervals $M /$ time step size $\Delta t$} \\
& $64 / \frac{1}{16}$ & $128 / \frac{1}{32}$ & $256 / \frac{1}{64}$ & $512 / \frac{1}{128}$ & $1024 / \frac{1}{256}$ \\
\hline$E_{10^{-6}, 10^{-7}}^{M, \Delta t}$ & $0.096949 \mathrm{e}-2$ & $0.049906 \mathrm{e}-2$ & $0.025231 \mathrm{e}-2$ & $0.012824 \mathrm{e}-2$ & $0.006446 \mathrm{e}-2$ \\
$r_{10^{-6}, 10^{-7}}^{M, \Delta t}$ & 0.95802 & 0.98400 & 0.97638 & 0.99233 & - \\
\hline$E_{10^{-7}, 10^{-7}}^{M, \Delta t}$ & $0.098712 \mathrm{e}-2$ & $0.050049 \mathrm{e}-2$ & $0.025485 \mathrm{e}-2$ & $0.012853 \mathrm{e}-2$ & $0.006449 \mathrm{e}-2$ \\
$r_{10^{-7}, 10^{-7}}^{M, \Delta t}$ & 0.97987 & 0.97368 & 0.98758 & 0.99493 & - \\
\hline$E_{10^{-8}, 10^{-7}}^{M, \Delta t}$ & $0.0951284 \mathrm{e}-2$ & $0.050026 \mathrm{e}-2$ & $0.025237 \mathrm{e}-2$ & $0.012781 \mathrm{e}-2$ & $0.006442 \mathrm{e}-2$ \\
$r_{10^{-8}, 10^{-7}}^{M, \Delta t}$ & 0.92720 & 0.98713 & 0.98156 & 0.98839 & - \\
\hline$E_{10^{-9}, 10^{-7}}^{M, \Delta t}$ & $0.096746 \mathrm{e}-2$ & $0.050012 \mathrm{e}-2$ & $0.025461 \mathrm{e}-2$ & $0.0128036 \mathrm{e}-2$ & $0.006437 \mathrm{e}-2$ \\
$r_{10^{-9}, 10^{-7}}^{M}$ & 0.95193 & 0.97394 & 0.99186 & 0.99188 & - \\
\hline Max. No. Iter. & 21 & 37 & 51 & 73 & 88 \\
\hline
\end{tabular}

Table 5: Maximum errors and orders of convergence for Example 4.2 over all time levels. 


\begin{tabular}{cccccc}
\hline & \multicolumn{6}{c}{ Number of space intervals $M /$ time step size $\Delta t$} \\
& $64 / \frac{1}{16}$ & $128 / \frac{1}{32}$ & $256 / \frac{1}{64}$ & $512 / \frac{1}{128}$ & $1024 / \frac{1}{256}$ \\
\hline$E_{\text {red }}^{M}$ & $0.96427 \mathrm{e}-3$ & $0.49949 \mathrm{e}-3$ & $0.25427 \mathrm{e}-3$ & $0.12830 \mathrm{e}-3$ & $0.06444 \mathrm{e}-3$ \\
$r_{\text {red }}^{M}$ & $0.94898 \mathrm{e}-3$ & $0.97404 \mathrm{e}-3$ & $0.98687 \mathrm{e}-3$ & $0.99336 \mathrm{e}-3$ & - \\
\hline
\end{tabular}

Table 6: Errors and orders of convergence for the reduced problem Example 4.2 over all time steps.

\begin{tabular}{cccccc}
\hline$(\varepsilon, \mu) \in \mathcal{P}$ & \multicolumn{5}{c}{ Number of space intervals $M /$ time step size $\Delta t$} \\
& $64 / \frac{1}{16}$ & $128 / \frac{1}{32}$ & $256 / \frac{1}{64}$ & $512 / \frac{1}{128}$ & $1024 / \frac{1}{256}$ \\
\hline$E_{E q u i}^{M}$ & $0.011318 \mathrm{e}-1$ & $0.05603 \mathrm{e}-2$ & $0.02634 \mathrm{e}-2$ & $0.01290 \mathrm{e}-2$ & $0.00648 \mathrm{e}-2$ \\
$r_{E q u i}^{M}$ & 1.0144 & 1.089 & 1.0300 & 0.9935 & - \\
\hline$E_{\text {Shish }}^{M}$ & $0.096427 \mathrm{e}-2$ & $0.049949 \mathrm{e}-2$ & $0.025427 \mathrm{e}-2$ & $0.012830 \mathrm{e}-2$ & $0.006446 \mathrm{e}-2$ \\
$r_{\text {Shish }}^{M}$ & 0.94898 & 0.97406 & 0.98687 & 0.99337 & - \\
\hline
\end{tabular}

Table 7: Uniform errors and orders of convergence for Example 4.2 over all time steps.

uniform errors between the Shishkin meshes and the proposed equidistributed meshes. These tables show that the order of convergence for the equidistributed mesh is better than that for Shishkin mesh. As a consequence, in general the uniform error obtained on the Shishkin mesh is larger than that on the equidistributed mesh. One can also note that a priori information of boundary layer location and its width is not required to generate the equidistributed mesh. From Table 6, one can see that the error $E_{r e d}^{M}$ and the order of convergence $r_{r e d}^{M}$ of the reduced problem (by taking $\varepsilon=0$ ) is also first-order accurate for the Example 4.2 .

The loglog error plot in Figure 1 (error vs $M$ plot) shows that the predicted order of convergence holds. The same predicted convergence order is observed also for Example 4.2, see Figure 3 (error vs $M$ plot). For this example, Figure 4 (each iteration vs $x$ plot) demonstrates the mesh movement towards the boundary points in each iteration. Figure 2 ( $x$ vs position of mesh points plot) for Example 4.1 shows that the layer adapted meshes are becoming dense towards the boundary points as the perturbation parameters $\varepsilon$ and $\mu$ are becoming small. In particular, the numerical experiments show that the parameter uniform convergence corresponding to both the parameters is ensured for the singularly perturbed parabolic IBVP as $M$ increases.

Remark 4.3 It should be noted, that the numerical experiments suggest that the condition $M^{-q} \leq C \Delta t$ with $0<q<1$ does not play any role in getting parameter uniform first-order accuracy of the proposed method.

\section{Conclusions}

In this paper, a computational method has been proposed for solving singularly perturbed parabolic initial boundary value problems for singularly perturbed $1 \mathrm{D}$ convection-diffusionreaction equations containing two small parameters. The proposed method uses the moving mesh technique by the equidistribution of a positive monitor function to generate the meshes. This method leads to first-order accurate numerical solution. A theoretical analysis is provided which shows that the numerical approximation is insensitive to the magnitude of the perturbation parameters. Numerical experiments illustrate the theoretical findings. 


\section{References}

[1] D. Arnold. A concise introduction to numerical analysis. Manuscript, 2001.

[2] M. G. Beckett and J. A. Mackenzie. Convergence analysis of finite difference approximations on equidistribted grids to a singularly perturbed boundary value problem. Appl. Numer. Math., 35:87-109, 2000.

[3] K. E. Brenan, S. L. Campbell, and L. R. Petzold. Numerical Solution of Initial-Value Problems in Differential Algebraic Equations. SIAM Publications, Philadelphia, PA, 2nd edition, 1996.

[4] C. Clavero, J.C. Jorge, and F. Lisbona. A uniformly convergent scheme on a nonuniform mesh for convection-diffusion parabolic problems. J. Comput. Appl. Math, 154:415-429, 2003.

[5] P. Das and S. Natesan. Richardson extrapolation method for singularly perturbed convection-diffusion problems on adaptively generated mesh. CMES Comput. Model. Eng. Sci., 90:463-485, 2013.

[6] M. El-Gamel. The sinc-Galerkin method for solving singularly perturbed reaction diffusion problem. Electron. Trans. Numer. Anal., 23:129-140, 2006.

[7] E. Emmrich. Stability and error of the variable two-step BDF for semilinear parabolic problems. J. Appl. Math. Comput., 19:33-55, 2005.

[8] E. Emmrich. Two-step BDF time discretisation of nonlinear evolution problems governed by monotone operators with strongly continuous perturbations. Comput. Methods Appl. Math., 9:37-62, 2009.

[9] E. Emmrich and R.D. Grigorieff. Supraconvergence of a finite difference scheme for elliptic boundary value problems of the third kind in fractional order sobolev spaces. Comput. Methods Appl. Math., 6:154-177, 2006.

[10] G. Evans, J. Blackledge, and P. Yardley. Numerical methods for partial differential equations. Springer, 2000.

[11] S. Gowrisankar and S. Natesan. The parameter uniform numerical method for singularly perturbed parabolic reaction diffusion problems on equidistributed grids. Appl. Math. Lett., 26:1053-1060, 2013.

[12] S. Gowrisankar and S. Natesan. Robust numerical scheme for singularly perturbed convection-diffusion parabolic initial-boundary-value problems on equidistributed grids. Comput. Phys. Commun., 185:2008 - 2019, 2014.

[13] E. Hairer and G. Wanner. Solving Ordinary Differential Equations II: Stiff and Differential-Algebraic Problems. Springer-Verlag, Berlin, Germany, 2nd edition, 1996.

[14] W. Huang, Y. Ren, and R. D. Russell. Moving mesh partial differential equations (MMPDES) based on the equidistribution principle. SIAM J. Numer. Anal., 31:709-730, 1994. 
[15] W. Huang and R. D. Russell. Adaptive moving mesh methods. Springer, 2011.

[16] M. K. Kadalbajoo and A. S. Yadaw. Parameter uniform finite element method for two parameter singularly perturbed parabolic reaction diffusion problems. Int. J. Comput. Meth., 9(4), 2012.

[17] N. Kopteva and M. Stynes. A robust adapive method for a quasilinear one-dimensional convection-diffusion problem. SIAM J. Numer. Anal., 39:1446-1467, 2001.

[18] D. Kumar. A computational technique for solving boundary value problems with two small parameters. Electron. J. Differential Equations, 30:1-30, 2013.

[19] P. Kunkel and V. Mehrmann. Differential-Algebraic Equations. Analysis and Numerical Solution. EMS Publishing House, Zürich, Switzerland, 2006.

[20] D. S. Naidu. Singular perturbations and time scales in control theory and applications: an overview. Dyn. Contin. Discrete Impuls. Syst. Ser. B Appl. Algorithms, 9(2):233-278, 2002.

[21] D. S. Naidu and A. Calise. Singular perturbations and time scales in guidance and control of aerospace systems: a survey. J. Guidance Control Dynam., 24(6):1057-1078, 2001.

[22] J. I. Ramos. An exponentially fitted methodnfor singularly perturbed one dimensional parabolic problems. Appl. Math. Comput., 161:513-525, 2005.

[23] J. Rashidinia, A. Barati, and M. Nabati. Appication of sinc- Galerkin method to singulary perturbed parabolic convection diffusion problems. Numer. Algorithms, 3:643-662, 2014.

[24] E. O. Riordan, M. L. Pickett, and G. I. Shishkin. Singularly perturbed problems modelling reaction-convection-diffusion processes. Comput. Methods Appl. Math., 3(3):424442, 2003.

[25] S. Sikwila and S. Shateyi. A moving mesh method for singularly perturbed problems. Abstr. Appl. Anal., 2013:1-11, 2013.

[26] R. S. Varga. Matrix iterative analysis. Springer, New York, 2nd edition, 2009.

[27] J. Vigo-Aguiar and S. Natesan. A parallel boundary value technique for singularly perturbed two-point boundary value problems. J. Supercomput., 27:195-206, 2004.

[28] J. Vigo-Aguiar and S. Natesan. An efficient numerical method for singular perturbation problems. J. Comput. Appl. Math., 192:132-141, 2006.

[29] X. Xu, W. Huang, R. D. Russell, and J. F. Williams. Convergence of de boors algorithm for the generation of equidistributing meshes. IMA J. Numer. Anal., 31:580-596, 2011.

[30] Y. Zhang, D. S. Naidu, C. Cai, and Y. Zou. Singular perturbations and time scales in control theory and applications: an overview 2002-2012. Int. J. Inf. Syst. Sci., 9(1):1-36, 2014. 